\title{
Características da atmosfera superior, a partir de dados de alta resolução obtidos à superfície
}

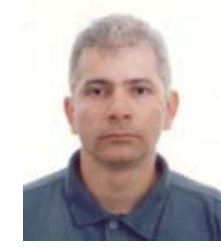

Enio P. Souza' ${ }^{1}$, Mário M. V. B. R. Leitão² \& Tatiane F. Barbosa ${ }^{3}$

\footnotetext{
1 DCA/UFPB, CEP 58109-970, Campina Grande, PB. Fone: (83) 310-1202. E-mail: esouza@dca.ufpb.br (Foto)

2 DCA/UFPB, CEP 58109-970, Campina Grande, PB. Fone: (83) 310-1202. E-mail: miranda@dca.ufpb.br

${ }^{3}$ Bolsista de Iniciação Científica do CNPq
}

Protocolo $098-27 / 6 / 2001$

\begin{abstract}
Resumo: Alguns parâmetros da atmosfera superior são calculados a partir de dados meteorológicos de superfície, coletados em alta resolução na cidade de Campina Grande, PB. Verificando-se, a cada dez minutos, os valores médios de temperatura, umidade e saldo de radiação, é possível ter-se uma idéia, nessa resolução temporal, de parâmetros importantes, tais como efeitos da cobertura de nuvem, altura da base das nuvens, energia potencial convectiva disponível e energia de inibição de convecção. Foi mostrado como a evolução desses parâmetros pode ser usada como ferramenta de análise meteorológica. A presença de nuvens altera bastante o valor dos parâmetros que podem ser estimados com dados de superfície.
\end{abstract}

Palavras-chave: dados da superfície, CAPE, tempo

\section{Characteristics of upper atmosphere from high-resolution surface data}

\begin{abstract}
Some parameters of the upper atmosphere are calculated from high-resolution surface data collected in the city of Campina Grande, PB. By verifying ten-minute mean values of temperature, moisture and radiation budget, it is possible to have an idea, in this time resolution, of parameters like: cloud cover effects, cloud base height, convective available potential energy and convection inhibition energy. It is shown how the evolution of these parameters can be used as a meteorological analysis tool. The presence of clouds changes the values of the parameters that can be estimated from surface data.
\end{abstract}

Key words: surface data, CAPE, weather

\section{INTRODUÇÃO}

Dados meteorológicos coletados em estações de superfície são utilizados, primeiramente, para auxiliar diretamente na previsão de tempo (numérica e subjetiva) e também como verdade terrestre na obtenção de perfis verticais, através de satélite, com a finalidade de serem usados em previsão numérica de tempo. Por outro lado, registros longos de dados de superfície podem ser usados em análises estatísticas do regime climático de um local ou região, porém, existem informações sobre a estrutura vertical da atmosfera, que podem ser inferidas a partir de dados de superfície, o que representa grande potencial tanto para o diagnóstico quanto para a previsão de tempo.

A partir de medições de pressão, temperatura e umidade do ar próximos à superfície, várias grandezas podem ser calculadas, dentre as quais algumas apresentam forte ligação com a estrutura vertical da atmosfera e podem ser utilizadas para uma compreensão melhor do seu status e evolução. Bolton
(1980) apresenta um conjunto de equações que podem ser usadas para cálculos mais precisos de temperatura potencial $(\theta)$, temperatura potencial equivalente $\left(\theta_{\mathrm{E}}\right)$, além de temperatura e pressão do nível de condensação por levantamento $T_{L}$ e $p_{L}$, respectivamente. Betts (1982 e 1985) apresenta uma teoria que relaciona a altura do nível de condensação por levantamento (NCL) às características do transporte de umidade e calor devido a nuvens convectivas.

A energia disponível para os processos atmosféricos é substanciada, em sua maior parte, pela disponibilidade de energia à superfície, como conseqüência do balanço de radiação. Por outro lado, vários parâmetros derivados dos dados de superfície são indicativos da situação na atmosfera superior. A altura do NCL, por exemplo, é um forte indicador da configuração da cobertura de nuvens convectivas (Wilde et al., 1985; Wetzel, 1990). A temperatura potencial equivalente, que equivale à entropia em um processo úmido, é um bom indicador da maneira pela qual se dará o ajuste convectivo da atmosfera (Emanuel, 1986). Sobre as regiões continentais, devido ao forte ciclo 
diurno, a energia potencial convectiva disponível (CAPE) é bem correlacionada com o ciclo da temperatura do bulbo úmido próximo à superfície (Williams \& Rennó, 1993).

Objetivou-se com este trabalho estudar as características da atmosfera superior que podem ser obtidas a partir de dados de alta resolução coletados em estações automáticas de superfície. Para isto, utilizaram-se dados de uma estação automática de superfície, com resolução de 10 min, para uma seqüência de quatro dias em Campina Grande, PB.

\section{MATERIAL E MÉTODOS}

\section{Dados}

Os dados utilizados neste trabalho foram coletados em fevereiro de 2000, na cidade de Campina Grande $\left(7,2^{\circ} \mathrm{S}, 35,9^{\circ} \mathrm{W}\right.$, $508 \mathrm{~m}$ ) usando-se um sistema automático de aquisição de dados, o qual foi programado para efetuar leituras a cada segundo e gerar médias a cada $10 \mathrm{~min}$. Inicialmente, esses dados eram armazenados na memória de um datalogger e, a cada dia, transferidos para um módulo de armazenamento e, posteriormente, para um computador.

Embora os dados tenham sido coletados durante um mês, a discussão neste trabalho correspondeu a um período de quatro dias, de 15 a 18 de fevereiro de 2000, a partir da 0 h (tempo local) do dia 15 de fevereiro.

Em adição, foram utilizados alguns dados de ar superior, coletados em Campina Grande, entre os dias 24 de março e 05 de abril de 1995, durante o Experimento de Meso-escala na Atmosfera do Sertão - EMAS.

\section{Metodologia}

As constantes termodinâmicas, como constante universal específica do gás (R), calor específico a pressão e volume constantes, $c_{p}$ e $c_{v}$, e calor latente de mudança de fase L, sofrem algumas mudanças na presença de vapor d'água. Portanto, para o ar úmido, essas "constantes" são função do conteúdo de vapor d'água atmosférico expresso na razão de mistura (r), dada por:

$$
\mathrm{r}=\varepsilon \frac{\mathrm{e}}{\mathrm{p}-\mathrm{e}}
$$

em que:

$\varepsilon \quad$ - razão entre as massas moleculares do vapor d'água e do ar seco, 0,622

e - pressão parcial do vapor d'água, $\mathrm{hPa}$

$\mathrm{p}$ - pressão atmosférica, $\mathrm{hPa}$

A razão de mistura é usualmente expressa em gramas de vapor por quilograma de ar seco, o que é feito multiplicando-se $\varepsilon$ por 1000 . O valor de saturação de razão de mistura $r_{s}$, pode ser obtido da Eq. (1) com a pressão de saturação do vapor $\mathrm{e}_{\mathrm{s}} \mathrm{em}$ lugar de e.

De acordo com Bolton (1980), a temperatura potencial do ar úmido pode ser dada por:

$$
\theta=T\left(\frac{\mathrm{p}_{0}}{\mathrm{p}}\right)^{0,2854\left(1-0,28 \times 10^{-3} \mathrm{r}\right)}
$$

em que:

$$
\mathrm{p}_{0} \quad \text { - pressão de referência, } 1000 \mathrm{hPa}
$$

O nível de condensação por levantamento (NCL) é o nível no qual uma parcela em ascensão adiabática atinge a saturação. Com os valores da temperatura de um nível $\mathrm{T}$ e da umidade relativa (UR), a temperatura do NCL, $\mathrm{T}_{\mathrm{L}}$, pode ser obtida da seguinte expressão, Bolton (1980):

$$
\mathrm{T}_{\mathrm{L}}=55,0+\frac{1}{\frac{1}{\mathrm{~T}-55}-\frac{\ln (\mathrm{UR} / 100)}{2840}}
$$

Portanto, a pressão do NCL $\mathrm{p}_{\mathrm{L}}$ pode ser obtida por:

$$
\mathrm{p}_{\mathrm{L}}=\mathrm{p}_{0}\left(\frac{\mathrm{T}_{\mathrm{L}}}{\theta}\right)^{1 /\left[0,2854\left(1-0,28 \times 10^{-3} \mathrm{r}\right)\right]}
$$

A partir da determinação da temperatura do NCL, a temperatura potencial equivalente $\theta_{\mathrm{E}}$ pode ser escrita na forma, (Bolton, 1980):

$$
\theta_{\mathrm{E}}=\theta \exp \left(\frac{2.675 \mathrm{r}}{\mathrm{T}_{\mathrm{L}}}\right)
$$

De forma semelhante, a temperatura potencial equivalente de saturação $\theta_{\mathrm{ES}}$ pode ser escrita como:

$$
\theta_{\mathrm{ES}}=\theta \exp \left(\frac{2.675 \mathrm{r}_{\mathrm{S}}}{\mathrm{T}}\right)
$$

O processo seguido por uma parcela adiabática saturada é descrito por uma variável conservativa que tanto pode ser a temperatura potencial equivalente quanto a temperatura potencial do bulbo úmido $\theta_{\mathrm{w}}$. Esta última pode ser obtida a partir de um processo interativo na forma (Iribarne \& Godson, 1981):

$$
\theta_{\mathrm{E}}=\theta_{\mathrm{W}}+\frac{\operatorname{Lr}_{\mathrm{s}}\left(\theta_{\mathrm{W}}\right)}{\mathrm{c}_{\mathrm{p}}}
$$

Uma função de bastante interesse na análise das condições da atmosfera, no tocante à sua propensão para formação de nuvens convectivas, é a energia potencial convectiva disponível - CAPE, a qual função representa o trabalho realizado por unidade de massa por uma parcela em ascensão com empuxo positivo. Na disponibilidade de dados de altitude, CAPE é definida, para um processo pseudo-adiabático, como (Williams \& Rennó, 1993):

$$
\mathrm{CAPE}=\mathrm{R} \int_{\mathrm{p}_{\mathrm{NEE}}}^{\mathrm{p}_{\mathrm{NCE}}}\left(\mathrm{T}_{\mathrm{vp}}-\mathrm{T}_{\mathrm{va}}\right) \mathrm{d} \ln \mathrm{p}
$$

em que:

$\mathrm{R}$ - constante universal para o ar seco, $\mathrm{J} \mathrm{kg}^{-1} \mathrm{~K}^{-1}$ 
$\mathrm{T}_{\mathrm{vp}}$ - temperatura virtual da parcela em ascensão, $\mathrm{K}$

$\mathrm{T}_{\text {va }}$ - temperatura virtual do ambiente, $\mathrm{K}$

NCE - nível de convecção espontânea

NPE - nível de perda de empuxo

Na Eq. (8) o valor da integral para dois níveis fixos de pressão depende dos valores das temperaturas virtuais da parcela e do ambiente. Enquanto a atmosfera se resfria de forma lenta (o balanço de radiação implica em um resfriamento da ordem de $1{ }^{\circ} \mathrm{C} \mathrm{d}^{-1}$, o que é compensado, em parte, por advecção), o ar próximo à superfície varia mais rapidamente, devido aos fluxos de entalpia e de umidade provenientes do solo, que sofre um forte ciclo durante o dia. Como conseqüência, a temperatura potencial do bulbo úmido à superfície varia bastante, implicando em um forte ciclo diário da temperatura virtual da parcela, na Eq. (8). Desta forma, o valor da CAPE pode ser obtido como função linear da temperatura potencial do bulbo úmido.

Williams \& Rennó (1993) estudaram vários conjuntos de dados sobre as áreas continental e oceânica da região tropical e concluíram que, do ponto de vista estatístico, CAPE pode ser determinada a partir da temperatura do bulbo úmido da superfície, com erro inferior a $25 \%$. Considerando-se como valor de CAPE-zero a temperatura potencial do bulbo úmido de 295,5 K (isto é: abaixo desse valor, não existe área de energia positiva em uma sondagem) com um aumento de $800 \mathrm{~J} \mathrm{~kg}^{-1}$ para cada grau de aquecimento (tomando-se como base algumas estações da região amazônica) o valor da CAPE pode ser obtido em função de $\theta_{\mathrm{W}}$ na forma:

$$
\mathrm{CAPE}=800\left(\theta_{\mathrm{W}}-295,5\right)
$$

Embora o valor da CAPE calculado pela Eq. (9) esteja sujeito a um erro, é possível se visualizar a evolução temporal dessa variável, com razoável precisão. Uma vez que a disponibilidade de sondagens atmosféricas é pequena (operacionalmente, essa informação pode ser obtida, no melhor dos casos, quatro vezes por dia) a possibilidade de se ter informação da CAPE a partir de dados de superfície consiste em uma boa ferramenta de análise das condições da atmosfera.

\section{RESULTADOS E DISCUSSÃO}

As figuras apresentadas a seguir correspondem aos quatro dias analisados, isto é, ao período de 15 a 18 de fevereiro de 2000 , a partir de $0 \mathrm{~h}$ do dia 15 . A Figura 1 mostra a evolução da temperatura potencial. Pode-se notar um forte ciclo diário com amplitude de aproximadamente $10 \mathrm{~K}$. Um aspecto interessante é a maneira como os dados de alta resolução captam mudanças rápidas de temperatura, o que se perde na suavização dos dados de baixa resolução. Essas variações rápidas podem estar relacionadas ao sombreamento, devido à presença de nuvens $\mathrm{e}$ a precipitação. Pode-se constatar a ocorrência de resfriamentos rápidos às $15 \mathrm{~h}$ no primeiro dia, às $14 \mathrm{~h}$ no segundo dia $(38 \mathrm{~h})$, às $19 \mathrm{~h}$ no segundo dia ( $43 \mathrm{~h})$ e às $15 \mathrm{~h}$ no terceiro dia $(63 \mathrm{~h})$. Será discutido, depois, quais desses resfriamentos estão relacionados com precipitação.

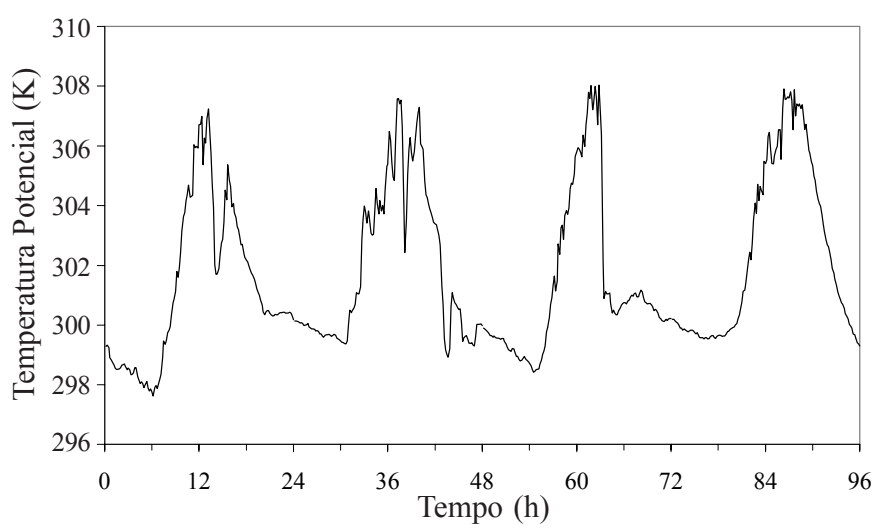

Figura 1. Evolução temporal da temperatura potencial

A evolução da razão de mistura é mostrada na Figura 2. Há, também, um claro ciclo diário. Da mesma maneira como ocorre com a temperatura, existem quedas bruscas na razão de mistura. A diminuição observada na temperatura, por volta das $15 \mathrm{~h}$ do primeiro dia, também é verificada na razão de mistura. Por outro lado, o resfriamento observado às $14 \mathrm{~h}$ do segundo dia, não corresponde à secagem. Note-se, também, que a razão de mistura é bastante baixa no último dia, comparando-se com os dias anteriores.

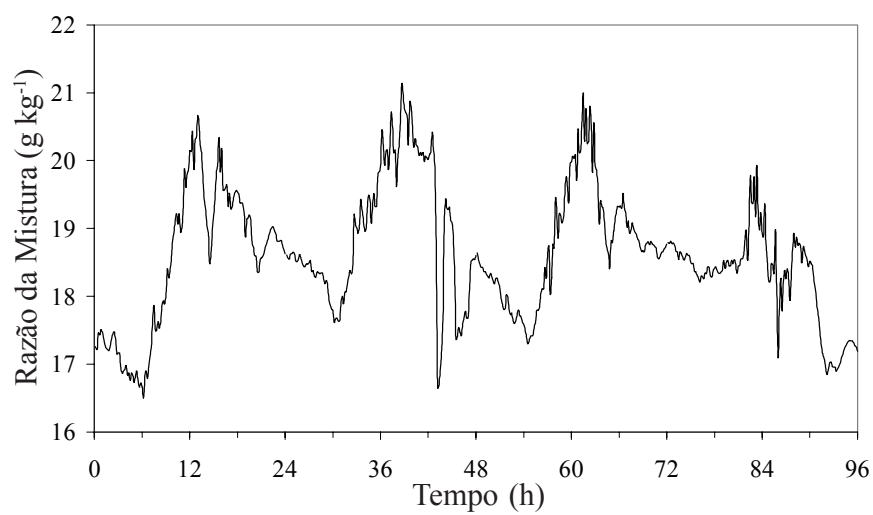

Figura 2. Evolução temporal da razão de mistura

Um aspecto interessante dos campos de razão de mistura e de temperatura é a boa correlação ente eles na escala de tempo inferior a uma hora. Além do efeito do sombreamento pelas nuvens, esse padrão aquecimento/umedecimento e resfriamento/secagem deve estar relacionado à atividade dos vórtices turbulentos de pequena escala, cujas partes ascendentes transportam ar mais quente e mais úmido, enquanto as partes descendentes transportam ar mais frio $\mathrm{e}$ mais seco.

Uma vez que as observações convencionais para o Nordeste do Brasil são realizadas às $9 \mathrm{e}$ às $15 \mathrm{~h}$, uma parte muito importante da sua evolução, que ocorre entre as duas observações, deixa de ser vista. Isto mostra a importância das estações automáticas de alta resolução temporal.

A Figura 3 indica a precipitação observada durante o período. Ocorreu uma pequena precipitação de $0,2 \mathrm{~mm}$ às $6 \mathrm{~h}$ do primeiro dia. A próxima chuva significativa ocorreu no segundo dia, às $14 \mathrm{~h}$, e foi de $3,1 \mathrm{~mm}$ em $10 \mathrm{~min}$, ou seja, $18,6 \mathrm{~mm} \mathrm{~h}^{-1}$. Às $19 \mathrm{~h}$ do segundo dia registraram-se $2,7 \mathrm{~mm}$ e $0,3 \mathrm{~mm}$ a zero hora do dia 17 (48 h). A partir das $15 \mathrm{~h}$ do dia 17 (63 h), choveu 3,5 mm em 
$2 \mathrm{~h}$. O último registro de precipitação ocorreu às $4 \mathrm{~h}$ do dia $18 \mathrm{e}$ foi de $1,7 \mathrm{~mm}$. Essa mesma figura pode ser utilizada para melhor compreensão de alguns aspectos das Figuras 1 e 2; por exemplo, o resfriamento e a secagem observados por volta das $15 \mathrm{~h}$ do primeiro dia devem estar relacionados a um forte sombreamento, já que têm duração de aproximadamente uma hora; entretanto, tal aspecto não esta relacionado com precipitação. Por volta das $38 \mathrm{~h}$ (14 h do dia 16) ocorreu o evento mais intenso de precipitação. A assinatura desse episódio pode ser vista através de um resfriamento/secagem nas Figuras 1 e 2. Note-se, entretanto, que a secagem não é tão intensa quanto o resfriamento, indicando que parte da precipitação pode ter-se reevaporado antes de atingir o solo.

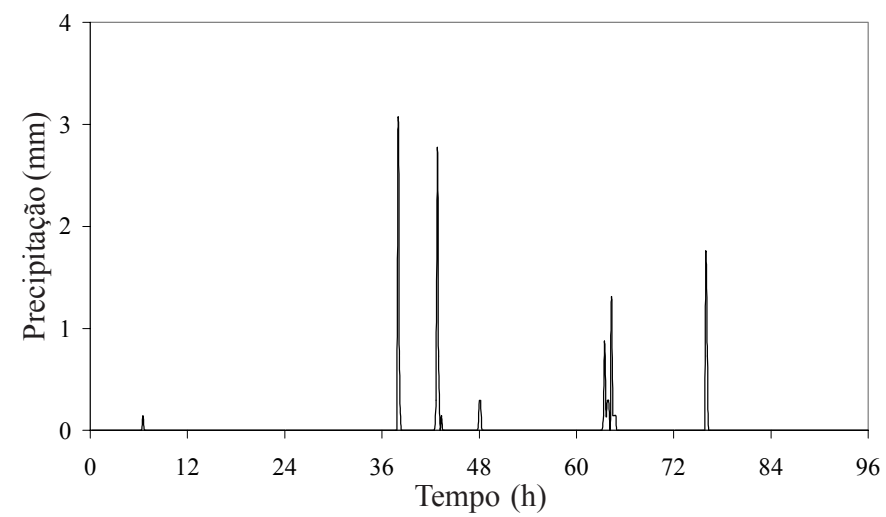

Figura 3. Precipitação observada em intervalos de 10 min

Característica diferente é observada em virtude do evento chuvoso das $43 \mathrm{~h}$ (19 h do dia 16) caso em que ocorre uma grande secagem. O resfriamento, porém, é inferior ao observado às $14 \mathrm{~h}$, indicando menor reevaporação. Outro evento importante de precipitação ocorreu entre 63 e 65 h (15 e 17 h do dia 17). O impacto sobre a temperatura foi marcante, reduzindo-a, às $15 \mathrm{~h}$, para valores tipicamente noturnos. No caso da razão de mistura, houve redução seguida de aumento. A precipitação ocorrida às $76 \mathrm{~h}$ (4 h do dia 18$)$ não afetou apreciavelmente a temperatura nem a umidade.

O saldo de radiação à superfície é o resultado de vários fatores, dentre eles a cobertura de nuvens, o conteúdo de umidade da atmosfera e as condições de superfície que determinam tanto a quantidade de radiação solar que será refletida quanto a quantidade de radiação terrestre que será emitida. O saldo de radiação no nível da estação, para o período, é mostrado na Figura 4. Embora a amplitude do ciclo diário se mantenha razoavelmente constante (em torno de $800 \mathrm{~W} \mathrm{~m}^{-2}$ ) a evolução é bastante diferente para cada dia. Não obstante, para cada dia o saldo de radiação se torna negativo depois das $18 \mathrm{~h}$.

Fica claro, por exemplo, que a secagem e o resfriamento que ocorreram às $14 \mathrm{~h}$ do primeiro dia são o resultado do aumento da cobertura de nuvens (que não causaram precipitação); tal cobertura deve ter sido causada por nuvem com espessura ótica grande o suficiente para reduzir o saldo de radiação para $10 \%$ do valor observado ao meio dia.

Durante a manhã do segundo dia, o saldo de radiação apresentou-se de forma oscilante entre $400 \mathrm{~W} \mathrm{~m}^{-2}$ e valores ligeiramente inferiores a $200 \mathrm{~W} \mathrm{~m}^{-2}$, que deve estar relacionado

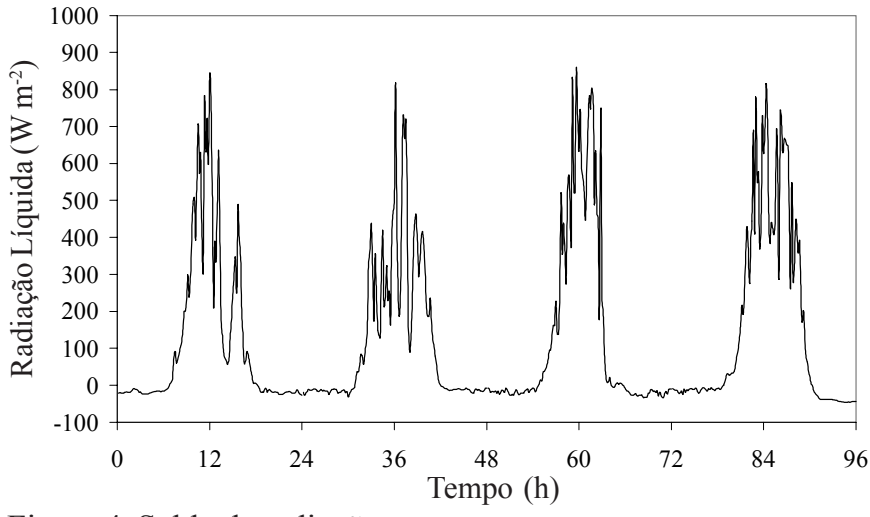

Figura 4. Saldo de radiação

à passagem de nuvens que sombrearam a estação, de forma a não permitir um aumento gradual da radiação solar a atingir o solo. O saldo de radiação só aumenta para o seu valor de pico por volta do meio-dia. $\mathrm{O}$ evento chuvoso ocorrido às $14 \mathrm{~h}$ do dia 16 contribuiu para uma redução drástica do saldo de radiação, como poderia ser esperado.

De forma semelhante, o evento chuvoso ocorrido na tarde do dia 17 levou o saldo de radiação para valores próximos de zero às $15 \mathrm{~h}$ desse dia. A partir desse horário, o saldo de radiação adquiriu características noturnas. $\mathrm{O}$ dia 18 foi o dia em que se observou o maior saldo de radiação. Embora a presença de nuvens seja evidente, a oscilação da curva mostra que o saldo de radiação esteve sempre retornando para o seu valor máximo. Esse é o único dia, dentre os quatro analisados, em que o saldo de radiação, no período da tarde, não é significativamente afetado pela presença de nuvens.

Serão mostrados, agora, alguns parâmetros da atmosfera superior que podem ser obtidos a partir das informações de superfície e que ajudam na compreensão dos sistemas de tempo que atuaram em Campina Grande, durante os dias analisados.

Dadas as condições da atmosfera, as parcelas em ascensão que terão mais chance de atingir a saturação e formar nuvens serão aquelas que possuírem maior empuxo e que possam atingir a condensação mais facilmente. De fato, Wilde et al. (1985) mostraram que a cobertura de nuvens cúmulos é condicionada pela diferença de altura entre a camada de entranhamento e o nível de condensação por levantamento - NCL. Por outro lado, Rennó \& Williams (1995) mostraram que as parcelas de ar mais propícias para atingirem condensação são aquelas que se formam próximo à superfície e adquirem energia suficiente para ascender e atingir o nível de saturação.

Quanto maior for a diferença entre a pressão em superfície e a do NCL, maior será também a altura do NCL e, portanto, menor tenderá a ser a cobertura de nuvens. Não se pode analisar, neste caso, a cobertura de nuvens em função do ciclo diário, porque as nuvens formadas durante o dia devem sua existência mais à convecção térmica, enquanto a noturna é mais devida à convecção mecânica (Stull, 1988). Esta última depende do cizalhamento vertical do vento, que não pode ser determinado a partir de dados de superfície. Não obstante, a variação da altura do NCL ao longo do período diurno, pode ser comparada com a evolução da cobertura de nuvens.

A Figura 5 mostra a evolução da diferença de pressão entre a superfície e o NCL, para o período. Para uma comparação 
entre a pressão e a altura, $100 \mathrm{hPa}$ nos primeiros níveis da vertical equivalem a aproximadamente $1 \mathrm{~km}$ de altura. De forma geral, a altura do NCL aumenta rapidamente no período da manhã. Como, tipicamente, a altura da zona de entranhamento evolui muito durante a manhã, a cobertura de nuvens induzidas termicamente começa a aumentar depois das $10 \mathrm{~h}$ da manhã (Wilde et al., 1985). A Figura 5 indica que, quando acontece diminuição da altura do NCL, existe diminuição do saldo de radiação (Figura 4) indicando aumento da cobertura de nuvens.

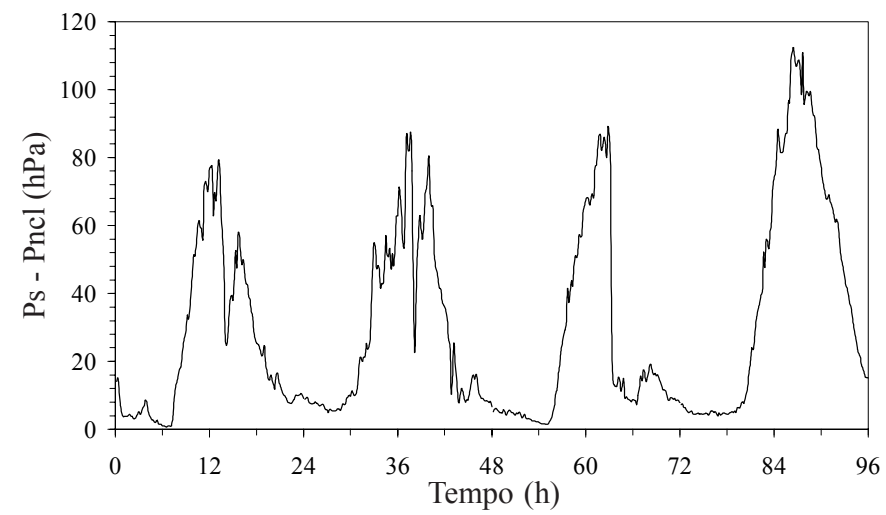

Figura 5. Evolução da diferença de pressão entre o NCL e a superfície

Comparando-se os dias 15 e 18, fica evidente que no dia 15 as condições para a formação de nuvens são mais favoráveis que no dia 18. A diferença de altura da base das nuvens entre os dois dias é de aproximadamente $300 \mathrm{~m}$, justificando o fato de o balanço de radiação ter sido maior no dia 18 , quando comparado ao balanço do dia 15 .

A Figura 6 indica a evolução da energia potencial convectiva disponível CAPE, calculada de acordo com a Eq. (9) CAPE apresenta um forte ciclo diário, como seria esperado sobre o continente. Comparando-se com a Figura 3, vê-se que os principais eventos chuvosos ocorrem em seguida a um grande aumento no valor da CAPE, mostrando que essa energia convectiva é inicialmente acumulada e liberada durante os eventos chuvosos. Note-se, também, que o valor da CAPE é reduzido depois de um evento chuvoso, indicando que a energia potencial convectiva gerada é consumida pela atividade dos cúmulos.

A comparação entre os quatro dias mostra que o valor máximo da CAPE no dia 18 (quarto dia) é menor que nos dias

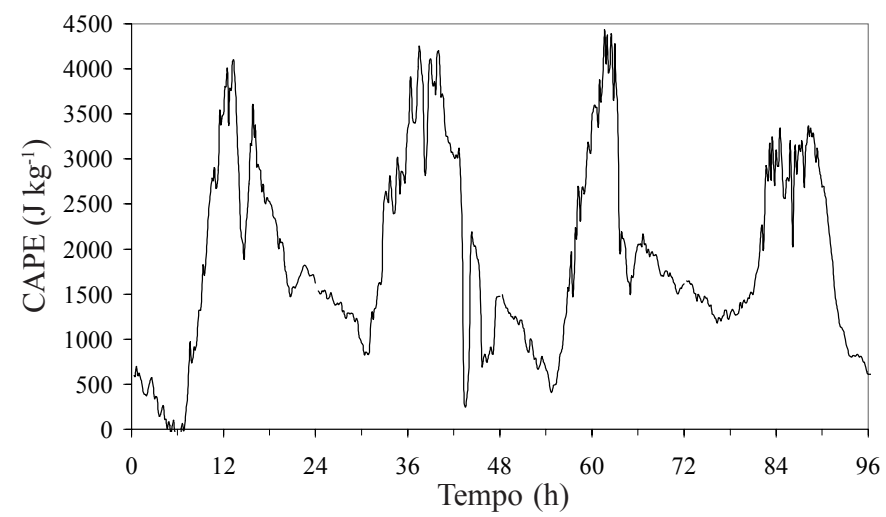

Figura 6. Evolução da energia potencial convectiva disponível (CAPE) anteriores. De fato, no dia 18 não se registrou chuva no período diurno, nem no dia 15 se registrou precipitação durante o dia; entretanto, tudo indica que nuvens espessas cobriam a região por volta das $14 \mathrm{~h}$ e isto mostra outra evidência apresentada por Williams \& Rennó (1993): a de que, mesmo na presença de um substancial valor da CAPE, nem sempre correspondem eventos chuvosos.

Os valores de CAPE calculados de acordo com a Eq. (9) são realísticos, podendo ser comprovados pela Tabela 1 , que mostra valores da CAPE calculados de acordo com a Eq. (8) para um conjunto de radiossondagens realizadas durante o Experimento de Meso-Escala na Atmosfera do Sertão - EMAS, que ocorreu entre os dias 24 de março e 05 de abril de 1995, em Campina Grande. Os dados apresentados correspondem às sondagens das $15 \mathrm{~h}$.

Tabela 1. Valores de CAPE às $15 \mathrm{~h}$, durante o experimento EMAS em Campina Grande, realizado nos meses de março e abril de 1995

\begin{tabular}{cccc}
\hline Dia/Mês & $\begin{array}{c}\text { CAPE } \\
\left(\mathrm{J} \mathrm{kg}^{-1}\right)\end{array}$ & Dia/Mês & $\begin{array}{c}\text { CAPE } \\
\left(\mathrm{J} \mathrm{kg}^{-1}\right)\end{array}$ \\
\hline 24/mar & 2993 & $30 /$ mar & - \\
25/mar & 3537 & $31 /$ mar & 3304 \\
26/mar & 2016 & $01 / \mathrm{abr}$ & 2188 \\
27/mar & 3062 & $02 / \mathrm{abr}$ & 1243 \\
28/mar & - & $03 / \mathrm{abr}$ & 2483 \\
29/mar & 612 & $04 / \mathrm{abr}$ & 2489 \\
\hline
\end{tabular}

De acordo com a Tabela 1, os valores da CAPE durante o dia podem ser tão pequenos quanto $612 \mathrm{~J} \mathrm{~kg}^{-1}$ (dia 29 de março) e tão grandes quanto $3537 \mathrm{~J} \mathrm{~kg}^{-1}$ (dia 25 de março); contudo, na maior parte dos casos o valor se situa entre 2000 e $3000 \mathrm{~J} \mathrm{~kg}^{-1}$, o que mostra que os valores da Figura 6, calculados pela Eq. (9) são realísticos.

A simples existência de CAPE na atmosfera não é condição suficiente para que ocorra a formação de nuvens e precipitação, visto que há outros fatores envolvidos no processo, que podem inibir a formação de nuvens convectivas, dentre eles o efeito do entranhamento de ar frio e seco nas parcelas em convecção e a energia de inibição, que deve ser vencida antes que as parcelas se tornem flutuantes (Williams \& Rennó, 1993). Este último fator é denominado energia convectiva de inibição CINE e corresponde à região dos primeiros níveis da atmosfera, na qual uma parcela em convecção está mais densa que o ar do ambiente e necessita, portanto, de um forçante externo para elevá-la até o nível de convecção espontânea - NCE. Na disponibilidade de sondagens atmosféricas, CINE pode ser obtida da Eq. (8) com a integração realizada entre o NCE e a superfície (Williams \& Rennó, 1993). É possível, contudo, ter-se uma idéia da quantidade de CINE da atmosfera a partir de dados de superfície, comparando-se os valores da temperatura potencial equivalente $\theta_{\mathrm{E}}$ e de saturação $\theta_{\mathrm{ES}}$ dados pelas Eqs. (5) e (6), respectivamente. Para esta comparação, pressupõe-se que o perfil vertical de $\theta_{\mathrm{ES}}$ é bem comportado, o que nem sempre é verdadeiro.

A Figura 7 mostra a evolução da diferença entre $\theta_{\mathrm{E}}$ e $\theta_{\mathrm{ES}}$ para o período. Pode-se ver que a inibição aumenta ao longo dos dias, sendo maior no último dia. Este padrão é coerente com o fato de ter havido menos nuvens no último dia. Note-se que, 
nos períodos nos quais há indicativo de atividade convectiva, o valor da inibição é pequeno, indicando que $\theta_{\mathrm{E}}-\theta_{\mathrm{E}}$ fornece uma indicação razoável da quantidade de inibição apresentada na atmosfera.

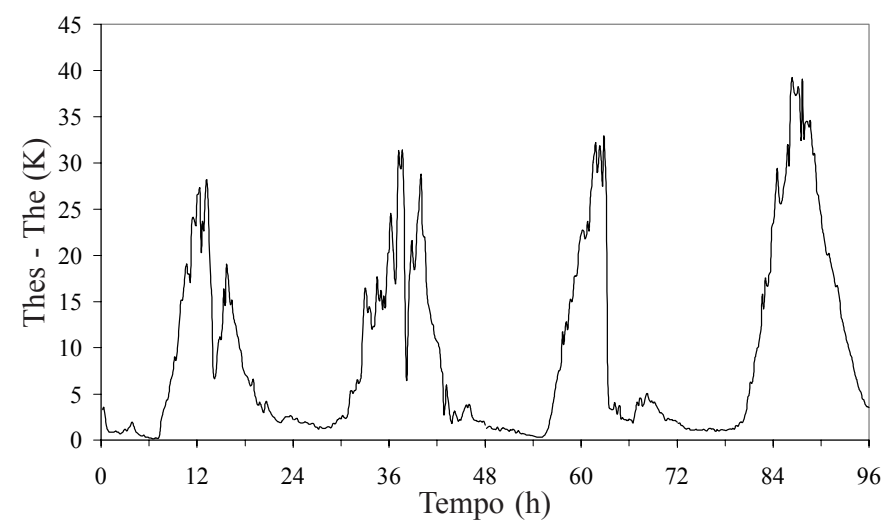

Figura 7. Evolução da diferença entre a temperatura potencial equivalente e a temperatura potencial equivalente de saturação

Os campos das Figuras 5 e 7 são bastante semelhantes, embora expressem grandezas diferentes. Ambos estão relacionados ao grau de saturação da atmosfera, mas dão idéia de processos distintos que ocorrem na atmosfera e que podem ser acessados a partir de dados de superfície e que, em alta resolução, oferecem visão mais concreta e ampla da evolução da atmosfera em uma escala de tempo inferior a uma hora.

A evolução de grandezas da atmosfera, obtidas a partir de dados de alta resolução, mostra aspectos importantes que ocorrem em escalas de tempo menores que as das observações convencionais e que podem explicar melhor os fenômenos de tempo que ocorreram sobre uma localidade, ao longo de certo período. Funções características da atmosfera superior podem ser obtidas a partir de dados de superfície e o conhecimento da evolução dessas funções é útil no diagnóstico do tempo.

\section{CONCLUSÕES}

1. Os dados de alta resolução revelam que, muitas vezes, o sombreamento por nuvens causa grande variação nos campos de temperatura e umidade. Isso tem fortes conseqüências na interpretação de dados de estações convencionais, coletados no máximo quatro vezes ao dia.

2. Dias com poucas nuvens são caracterizados por forte energia de inibição e NCL mais alto, em comparação aos dias nublados.
3. A precipitação diurna é precedida de um grande aumento da energia potencial convectiva disponível - CAPE, e isto é um importante ponto para investigação futura, com vistas ao seu uso como ferramenta preditiva.

\section{AGRADECIMENTOS}

Os autores agradecem à Universidade Federal da Paraíba (UFPB) e ao Conselho Nacional de Desenvolvimento Científico e Tecnológico (CNPq) pelo suporte na execução deste trabalho. Além do mais, os autores agradecem aos dois revisores anônimos, cujas sugestões contribuíram para uma melhoria do texto original.

\section{LITERATURA CITADA}

Betts, A. K. Saturation point analysis of moist convective overturning. Journal of the Atmospheric Sciences, Boston, v.39, p.1934-1945. 1982.

Betts, A. K. Mixing line analysis of clouds and cloudy boundary layers. Journal of the Atmospheric Sciences, Boston, v.42, p.2751-2763. 1985.

Bolton, D. The computation of equivalent potential temperature. Monthly Weather Review, Boston, v.108, p.1046-1053. 1980.

Emanuel, K. A. An air-sea interaction theory for tropical cyclones. Part I: Steady-state maintenance. Journal of the Atmospheric Sciences, Boston, v.43, p.585-604. 1986.

Iribarne, J. V.; Godson, W. L. Atmospheric thermodynamics. Dordrecht, The Netherlands: D. Reidel Publishing Co., 1981, 259p.

Rennó, N. O.; Williams, E., Quasi-Lagrangian measurements in convective boundary layer plums and their implications for calculation of CAPE. Monthly Weather Review, Boston, v.123, p.2733-2742. 1995.

Stull, R. B., An introduction to boundary layer meteorology. Dordrecht, The Netherlands: Kluwer Academic Publishers, 1988, 666p.

Wetzel, P. J. A simple parcel method for prediction of cumulus onset and area-averaged cloud amount over heterogeneous land surfaces. Journal of Applied Meteorology, Boston, v.29, p.516-523. 1990.

Wilde, N. P.; Stull, R. B.; Eloranta, E. W. The LCL zone and cumulus onset. Journal of Climate and Applied Meteorology, Boston, v.24, p.640-657. 1985.

Williams, E.; Rennó, N. An analysis of the conditional instability of the tropical atmosphere. Monthly Weather Review, Boston, v.121, p.21-36. 1993. 